\title{
Multistage Receiver for Differential Space-Time Block Codes
}

\author{
Poramate Tarasak \\ Department of Electrical \\ and Computer Engineering \\ University of Victoria, \\ P.O. Box 3055 STN CSC \\ Victoria, B.C., V8W 3P6, Canada \\ email: ptarasak@ece.uvic.ca
}

\author{
Hlaing Minn \\ Department of Electrical Engineering \\ Eric Jonsson School of Engineering \\ and Computer Science \\ University of Texas at Dallas \\ P.O. Box 830688, Mail Station EC33 \\ Richardson, TX 75083-0688, U.S.A. \\ email: hlaing.minn@utdallas.edu
}

\author{
Vijay K. Bhargava \\ Department of Electrical \\ and Computer Engineering \\ University of Victoria, \\ P.O. Box 3055 STN CSC \\ Victoria, B.C., V8W 3P6, Canada \\ email: bhargava@ece.uvic.ca
}

\begin{abstract}
Tarokh's differential space-time block codes (DSTBC) can provide full spatial diversity and can be decoded without channel state information. The bit error rate performance of a conventional differential detection has 3-dB degradation compared to a coherent detection in a quasi-static fading channel. Differential detection experiences more degradation in a fast fading channel and error floor appears at high SNR. In [5], an approximate maximum likelihood receiver was derived and it reduced the error floor significantly. In this paper, we modify the receiver in [5] and apply the concept of multistage decision-directed estimator with intrablock-interference cancellation to obtain further improvement. The proposed receiver works efficiently at moderate to high SNR and at the fading rate between $f_{d} T=0.02$ and $f_{d} T=0.07$.
\end{abstract}

\section{INTRODUCTION}

Space-time block codes [1] can provide full spatial diversity while channel coefficients have to be known at the receiver. In some situation such as when the mobile moves fast, the channel varies rapidly Then, accurate channel estimation becomes very difficult. Also, the receiver needs to estimate the channel for each transmit and receive antenna pair. This increases overhead and complexity. Differential space-time coding/modulation was proposed to avoid channel estimation. It can be classified as group [3], [4] and nongroup differential schemes [2]. Group design differential space-time modulation (DSTM) has the advantage that constellation is preserved and matrix multiplication in differential encoding can be avoided. Tarokh's differential space-time block codes (DSTBC) [2] is a popular nongroup differential scheme. Its advantage is a lower decoding complexity due to the orthogonal transmission matrix (Alamouti's scheme).

Conventional differential detection (DD) of DSTBC assumes fixed channel coefficients during two consecutive transmission blocks. It has about $3-\mathrm{dB}$ performance degradation compared to coherent detection with perfect channel state information. However, if the channel is fast-fading, conventional DD experiences more degradation due to the invalid assumption above. DD performance yields an irreducible error floor at moderate to high SNR region [5].
Recently, a few improved detection schemes over conventional DD have been proposed for DSTM and DSTBC to cope with time-varying channel characteristic. Optimal detection of DSTM applying multiple symbol detection and suboptimal detection applying decision-feedback differential detection were derived in [7]. The receiver provides large performance gains over conventional DD while the complexity increases moderately. A disadvantage is that the receiver needs to compute prediction coefficients according to channel and noise statistics. Another suboptimal detector of DSTM was derived in [8] where the prediction coefficients are designed without any knowledge of the channel and noise statistics. It performs close to the optimal detector when maximum normalized (by signaling interval) Doppler frequency is low $(\sim 0.01)$. Both studies consider DSTM signal with diagonal structure because it yields a simple decision rule. For DSTBC, an approximate maximum-likelihood (ML) detector was derived in [5]. The receiver outperforms conventional DD significantly. It reduces the error floor at least an order of magnitude.

In this paper, we concentrate on Tarokh's DSTBC [2] for BPSK constellation. The receiver proposed in [5] is modified to compensate for the assumption of fixed channel coefficients during one transmission block. Multistage receiver is proposed for DSTBC. In the first iteration, the same receiver as in [5] is applied. After the first iteration, the receiver computes channel estimate from the tentative decision. This channel estimate will be exploited in the modified detector to subtract intrablockinterference (IBI) in the second iteration. The idea of iterative use of channel estimate and symbol detection has been applied with multistage decision-directed receiver in DS-CDMA system [6]. However, the scenario here is quite different and the cancellation part is new. Multistage receiver is shown to reduce the error floor significantly, especially in fast-fading channel case. Both uncoded and coded system will be treated. The paper is organized as follows. Section II describes system model and notations. Section III reviews conventional DD and the receiver in [5]. Section IV proposes the Viterbi receiver with IBI cancellation. Section V discusses multistage receiver in which the proposed detector is applied. Section VI shows 
the simulation results and discussion. Finally, conclusions are given in section VII.

\section{SySTEM MODEL}

The system model and notations follow from [5]. We consider two transmit antennas and one receive antenna. An information vector $\boldsymbol{d}_{n}=\left[d_{n}^{1} d_{n}^{2}\right]^{t}$, which represents $n^{t h}$ pair of symbol, is selected from an $M$-ary $\left(2^{b}\right.$-ary) constellation according to the $2 b$ information bits. Each symbol has an amplitude of $1 / \sqrt{2}$ so that the average total transmit power from two transmit antennas is one (computed from (1) and (2). The vector $\boldsymbol{d}_{n}$ is multiplied by a unitary matrix to obtain a mapping vector

$$
\boldsymbol{x}_{n}=\left[\begin{array}{l}
A_{n} \\
B_{n}
\end{array}\right]=\frac{1}{\sqrt{2}}\left[\begin{array}{cc}
1 & 1 \\
-1 & 1
\end{array}\right] \boldsymbol{d}_{n} .
$$

Then, differential encoding produces a transmission vector [2]

$$
\boldsymbol{s}_{n}=\left[\begin{array}{c}
s_{2 n-1} \\
s_{2 n}
\end{array}\right]=\boldsymbol{D}_{n-1}^{t} \boldsymbol{x}_{n}
$$

where $\boldsymbol{D}_{n}=\left[\begin{array}{cc}s_{2 n-1} & s_{2 n} \\ -s_{2 n}^{*} & s_{2 n-1}^{*}\end{array}\right]$ is an Alamouti's transmission matrix. The symbol $s_{2 n-1}$ and $s_{2 n}$ are transmitted at signaling interval $2 n-1$ from the first and second antennas, respectively. The symbol $-s_{2 n}^{*}$ and $s_{2 n-1}^{*}$ are transmitted at signaling interval $2 n$ from the first and second antennas, respectively.

BPSK constellation is chosen for simplicity. Therefore, the system achieve $1 \mathrm{bit} / \mathrm{sec} / \mathrm{Hz}$ transmission rate. For higher modulation scheme, the approach in [5] and in this paper can still be applied. However, the complexity of the receiver will be higher due to constellation expansion.

The channel is frequency-flat Rayleigh fading with timevarying characteristic according to Jake's power spectrum. Let $a_{i}[n]$ denote a fading process from $i^{t h}$ transmit antenna, $i=$ 1,2 . Then, $a_{1}[n]$ and $a_{2}[n]$ are zero mean complex Gaussian random processes such that

- $a_{1}[n]$ and $a_{2}[n]$ are two independent Gaussian processes. This corresponds to the case when there is enough separation between transmit antennas so that the signal paths are independently affected by the channel.

- Their autocorrelation follows $R_{a}[m]=J_{0}\left(2 \pi f_{d} T m\right)$ where $J_{0}(\cdot)$ is the zeroth-order Bessel function of the first kind, $f_{d}$ is the maximum Doppler frequency, and $T$ is the signaling interval.

The detector in [5] assumes constant fading coefficients within a transmission block, i.e., during the $(2 n-1)^{t h}$ and $(2 n)^{t h}$ signaling intervals. Therefore, the received signal vector $\boldsymbol{r}_{n}=\left[\begin{array}{ll}r_{2 n-1} & r_{2 n}\end{array}\right]^{t}$ can be written as [5]

$$
\boldsymbol{r}_{n}=\boldsymbol{D}_{n} \boldsymbol{a}_{n}+\boldsymbol{w}_{n}
$$

where $\boldsymbol{a}_{n}=\left[a_{1}[2 n-1] a_{2}[2 n-1]\right]^{t}$ and $\boldsymbol{w}_{n}=\left[w_{2 n-1} w_{2 n}\right]^{t}$ represents a noise vector. The elements $w_{2 n-1}$ and $w_{2 n}$ are zero mean complex Gaussian random variables, each with variance $1 /(2 \mathrm{SNR})$ per dimension.

\section{Conventional Receiver And Approximate ML RECEIVER}

Differential detector of DSTBC assumes fixed channel coefficients during two consecutive transmission blocks. It is known that differential detector is optimal for a quasistatic channel [5]. The detector computes a vector $\boldsymbol{z}_{n+1}=$ $\left[z_{n+1}^{1} z_{n+1}^{2}\right]^{t}$, with $z_{n+1}^{1}=r[2 n+1] r^{*}[2 n-1]+r^{*}[2 n+2] r[2 n]$ and $z_{n+1}^{2}=r[2 n+1] r^{*}[2 n]-r^{*}[2 n+2] r[2 n-1]$. The decision rule of differential detector is [2]

$$
\hat{\boldsymbol{d}}_{n+1}=\underset{\boldsymbol{d}_{n+1}}{\arg \min }\left\|\boldsymbol{z}_{n+1}-\boldsymbol{x}_{n+1}\right\|^{2} .
$$

This detector will be noted as a conventional receiver (CR) [5].

For a time-varying channel, optimal detector is different from CR. Following [5] and assuming fixed channel during a transmission block, an approximate ML sequence detection will be summarized here.

Let the data sequence $\boldsymbol{d}=\left[\begin{array}{lll}\boldsymbol{d}_{1}^{t} & \boldsymbol{d}_{2}^{t} \ldots \boldsymbol{d}_{N-1}^{t}\end{array}\right]^{t}$, which corresponds to $s=\left[\begin{array}{llll}s_{0}^{t} & s_{1}^{t} \ldots s_{N-1}^{t}\end{array}\right]^{t}$ (given $s_{0}$ ) and the received sequence $\boldsymbol{r}=\left[\boldsymbol{r}_{0}^{t} \boldsymbol{r}_{1}^{t} \ldots \boldsymbol{r}_{N}^{t}\right]^{t}$. With the assumption of fixed fading gains during one transmission block, the receive sequence $\boldsymbol{r}$ can be expressed as $\boldsymbol{r}=\boldsymbol{D} \boldsymbol{a}+\boldsymbol{n}$ where $\boldsymbol{D}=$ $\operatorname{diag}\left\{\boldsymbol{D}_{n}, n=0,1, \ldots, N-1\right\}, \boldsymbol{a}=\left[\boldsymbol{a}_{0}^{t} \boldsymbol{a}_{1}^{t} \ldots \boldsymbol{a}_{N-1}^{t}\right]^{t}$ and $\boldsymbol{w}=\left[\boldsymbol{w}_{0}^{t} \boldsymbol{w}_{1}^{t} \ldots \boldsymbol{w}_{N-1}^{t}\right]^{t}$.

Due to one-to-one correspondence between $\boldsymbol{d}$ and $s$, the conditional probability $f\left(\boldsymbol{r} \mid \boldsymbol{d}, \boldsymbol{s}_{0}\right)$ is equivalent to $f(\boldsymbol{r} \mid \boldsymbol{s})$. Using a maximum-likelihood criterion, the ML receiver finds the sequence $\tilde{\boldsymbol{d}}=\left[\tilde{\boldsymbol{d}}_{1}^{t} \tilde{\boldsymbol{d}}_{2}^{t} \ldots \tilde{\boldsymbol{d}}_{N-1}^{t}\right]^{t}$ which satisfies

$$
\tilde{\boldsymbol{d}}=\arg \max _{\boldsymbol{d}} \log f(\boldsymbol{r} \mid \boldsymbol{s})
$$

where $f(\boldsymbol{r} \mid \boldsymbol{s})$ is the conditional probability density function (p.d.f.) of the vector $\boldsymbol{r}$ given $\boldsymbol{s}$. It is Gaussian distributed with zero mean and covariance $\boldsymbol{C}_{r \mid s}=\boldsymbol{D}\left(\boldsymbol{C}_{a}+\sigma_{w}^{2} \boldsymbol{I}_{2 N}\right) \boldsymbol{D}^{H}$ where $\boldsymbol{C}_{a}=E\left[\boldsymbol{a a ^ { H }}\right]$ is the fading covariance matrix [5] and $\boldsymbol{I}_{2 N}$ is a $2 N \times 2 N$ identity matrix. The log-likelihood function can be written as $l(\boldsymbol{r} \mid \boldsymbol{s})=-\boldsymbol{r}^{H} \boldsymbol{D} \boldsymbol{M}^{-1} \boldsymbol{D}^{H} \boldsymbol{r}$, where $\boldsymbol{M}=\boldsymbol{C}_{a}+\sigma_{w}^{2} \boldsymbol{I}_{2 N}$. Applying $L D L^{t}$ decomposition [12] to the inverse of the covariance matrix $\boldsymbol{M}$ obtains coefficients of the linear predictor of the process $a=$ $\left[a_{1}[-1] a_{2}[-1] a_{1}[1] a_{2}[1] \ldots a_{1}[2 N-3] a_{2}[2 N-3]\right]$. Because the process $\boldsymbol{a}_{1}[n]$ and $\boldsymbol{a}_{2}[n]$ are independent and identically distributed, the prediction coefficients are scalar and identical for both processes. Consequently, the prediction coefficients can be computed from $L D L^{t}$ decomposition of the matrix $\tilde{\boldsymbol{M}}^{-1}=\left(\boldsymbol{C}_{\dot{a}}+\sigma_{w}^{2} \boldsymbol{I}_{N}\right)^{-1}$ where $\boldsymbol{C}_{\dot{a}}=E\left[\dot{\boldsymbol{a}} \dot{\boldsymbol{a}}^{H}\right]$ and $\dot{\boldsymbol{a}}=$ $\left[a_{1}[-1] a_{1}[1] \ldots a_{1}[2 N-3]\right]^{t}$. Therefore, we have $\tilde{\boldsymbol{M}}^{-1}=$ $\boldsymbol{B}^{H} \Xi \boldsymbol{B}$ where

$$
\boldsymbol{B}=\left[\begin{array}{ccccc}
-b_{0}^{0} & 0 & 0 & \cdots & 0 \\
-b_{1}^{1} & -b_{0}^{1} & 0 & \ldots & 0 \\
-b_{2}^{2} & -b_{1}^{2} & -b_{0}^{2} & \ldots & 0 \\
\cdots & \cdots & \cdots & \cdots & \cdots \\
-b_{N-1}^{N-1} & -b_{N-2}^{N-1} & -b_{N-3}^{N-1} & \cdots & -b_{0}^{N-1}
\end{array}\right],
$$


$\Xi$ is a diagonal matrix containing inversed mean square values of prediction error as diagonal elements. The element $b_{0}^{i}=-1$ and $b_{j}^{i}$ is the $j^{t h}$ coefficient of the $i^{t h}$ order linear predictor for the random process $\boldsymbol{y}_{n}=\boldsymbol{D}_{n}^{H} \boldsymbol{r}_{n}=\boldsymbol{a}_{n}+\boldsymbol{w}_{n}$ [5]. The stationary process $\boldsymbol{y}_{n}$ can be represented as an autoregressive (AR) process generated by a linear predictor with suitable order $Q \ll N-1$. Using $L D L^{t}$ decomposition of $\boldsymbol{M}^{-1}$, the log-likelihood function can be readily written as

$$
l(\boldsymbol{r} \mid \boldsymbol{s})=-\sum_{n=0}^{N-1}\left\|\boldsymbol{D}_{n}^{H} \boldsymbol{r}_{n}-\hat{\boldsymbol{a}}_{n}\right\|^{2} / \xi_{n}
$$

where $\hat{\boldsymbol{a}}_{n}=\sum_{k=1}^{Q} b_{k}^{Q} \boldsymbol{D}_{n-k}^{H} \boldsymbol{r}_{n-k}$ represents the $Q^{t h}$ order prediction of $\boldsymbol{a}$. Now, the detection problem can be stated as to find the data sequence such that it minimizes the square error between the channel gains computed from the received signals and the predicted values. Similar approach has been applied successfully in [9], [10] with CPM and PSK, respectively.

The trellis structure of DSTBC can be defined as follows. Each trellis interval corresponds to a transmission matrix $\boldsymbol{D}_{n}$. For BPSK, the trellis consists of $2^{2 Q}$ states with $2^{2}$ emerging from each state and terminating at each state. Each state represents a $Q$-couple transmitted vector $\Gamma_{n}=\left[\begin{array}{llll}\boldsymbol{s}_{n} & \boldsymbol{s}_{n-1} & \ldots & \boldsymbol{s}_{n-Q+1}\end{array}\right]$. The transition from the state $\left[\boldsymbol{s}_{n} \boldsymbol{s}_{n-1} \ldots \boldsymbol{s}_{n-Q+1}\right]$ to $\left[\boldsymbol{s}_{n+1} \boldsymbol{s}_{n} \ldots \boldsymbol{s}_{n-Q+2}\right]$ is determined by differential encoding from (2). The branch metric associated with each transition can be defined as [5]

$$
\Lambda\left(\Gamma_{n}, \boldsymbol{d}_{n+1}\right)=\left\|\boldsymbol{D}_{n+1}^{H} \boldsymbol{r}_{n+1}-\sum_{k=1}^{Q} b_{k}^{Q} \boldsymbol{D}_{n+1-k}^{H} \boldsymbol{r}_{n+1-k}\right\|^{2} .
$$

Exploiting per-survivor processing technique, the number of states can be reduced to $2^{2 P}$ with $P<Q$. Now, the branch metric $\Lambda\left(\Gamma_{n}, \boldsymbol{d}_{n+1}\right)$ is determined from symbols associated with the transition and symbols along the survivor path terminating at the state $\Gamma_{n}$. The detection algorithm described above is referred to Viterbi receiver (VR). Note that this receiver requires knowledge of Doppler frequency and SNR which are assumed to be perfectly known at the receiver. In practice, some forms of Doppler frequency and SNR estimation have to be done and there will be errors between the actual values and the estimated ones.

\section{VITERBI RECEIVER WITH INTRABLOCK-INTERFERENCE CANCELLATION}

The Viterbi receiver described in the previous sections works well under the condition of fixed or very slowly varying channel gains during one transmission block. Now let us relax this condition and allow the channel to vary within a transmission block. The elements of the received vector $\boldsymbol{r}_{n}$ can be written as

$$
\begin{aligned}
r_{2 n-1} & =s_{2 n-1} a_{1}[2 n-1]+s_{2 n} a_{2}[2 n-1]+w_{2 n-1}, \\
r_{2 n} & =-s_{2 n}^{*} a_{1}[2 n]+s_{2 n-1}^{*} a_{2}[2 n]+w_{2 n}
\end{aligned}
$$

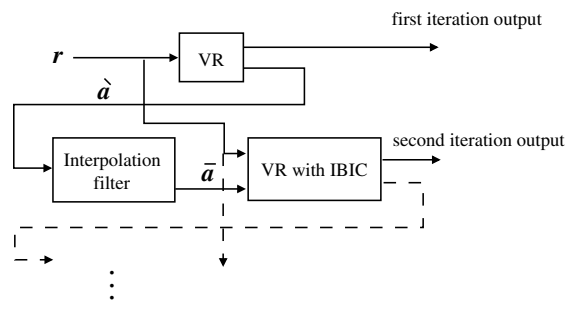

Fig. 1. Multistage detector for DSTBC

Defining $\Delta a_{1}=a_{1}[2 n]-a_{1}[2 n-1]$ and $\Delta a_{2}=a_{2}[2 n]-$ $a_{2}[2 n-1]$ and substituting them into $r_{2 n}$ in (9), we have

$$
\begin{aligned}
r_{2 n}=- & s_{2 n}^{*}\left(a_{1}[2 n-1]+\Delta a_{1}\right)+s_{2 n-1}^{*}\left(a_{2}[2 n-1]\right. \\
& \left.+\Delta a_{2}\right)+w_{2 n}
\end{aligned}
$$

Determining $\boldsymbol{D}_{n}^{H} \boldsymbol{r}_{n}$ from (9), (10) obtains the channel gains

$$
\begin{aligned}
\grave{a}_{1}[2 n-1] & =s_{2 n-1}^{*} r_{2 n-1}-s_{2 n} r_{2 n} \\
& =a_{1}[2 n-1]+I 1_{n}+s_{2 n-1}^{*} w_{2 n-1}-s_{2 n} w_{2 n} \\
\grave{a}_{2}[2 n-1] & =s_{2 n}^{*} r_{2 n-1}+s_{2 n-1} r_{2 n} \\
& =a_{2}[2 n-1]+I 2_{n}+s_{2 n}^{*} w_{2 n-1}+s_{2 n-1} w_{2 n}
\end{aligned}
$$

where $I 1_{n}=0.5 \Delta a_{1}-\Delta a_{2} s_{2 n-1}^{*} s_{2 n}$ and $I 2_{n}=0.5 \Delta a_{2}$ $\Delta a_{1} s_{2 n-1} s_{2 n}^{*}$ represent intrablock-interference (IBI). This means that even if we have correct transmission block $\boldsymbol{D}_{n}$, the channel gains are affected by IBI. The amount of IBI depends on how rapid the channel varies. At higher fading rate, the average power of IBI is higher. If the channel is fixed during one transmission block, $I 1_{n}, I 2_{n}$ are zero and (11) reduces to (3). The receiver can compensate for this IBI if it has some fading information. Then, it can subtract IBI using appropriate $\left[s_{2 n-1} s_{2 n}\right]^{t}$. This detection algorithm, which accounts for IBI, will be dubbed as Viterbi receiver with intrablock-interference cancellation (VR-IBIC).

\section{Multistage Receiver}

Multistage receiver composes of VR, interpolation filter and VR-IBIC as in Fig. 1. In the first iteration, VR performs ML detection as in [5]. However, as Viterbi algorithm performs along the trellis, it stores not only the decoded bit $d_{n}$, which is a tentative decision, but also $\left[\grave{a}_{1}[2 n-1] \grave{a}_{2}[2 n-\right.$ 1]] computed from (11) associated with each survivor path. VR provides sequence $\grave{\boldsymbol{a}}_{1}, \grave{\boldsymbol{a}}_{2}=\left[\grave{a}_{1}[-1] \grave{a}_{1}[1] \ldots \grave{a}_{1}[2 N-\right.$ $3]],\left[\grave{a}_{2}[-1] \grave{a}_{2}[1] \ldots \grave{a}_{2}[2 N-3]\right]$ to the second iteration of DSTBC decoding.

In the second iteration, the sequences $\grave{\boldsymbol{a}}_{1}, \grave{\boldsymbol{a}}_{2}$ are passed to the interpolation filter. The interpolation filter performs two functions. One is to obtain the channel estimate at even duration and the other is to filter out noise whose spectrum is outside the maximum Doppler frequency. Interpolation filter sampling rate is the same as signaling rate. The sequences $\grave{\boldsymbol{a}}_{1}, \grave{\boldsymbol{a}}_{2}$, which are at block rate, are padded with zero between 
samples before being filtered. The output of the filter $\overline{\boldsymbol{a}}_{1}, \overline{\boldsymbol{a}}_{2}$ act as channel estimates to be employed in VR-IBIC.

VR-IBIC computes $\Delta \bar{a}_{1}=\bar{a}_{1}[2 n]-\bar{a}_{1}[2 n-1]$ and $\Delta \bar{a}_{2}=\bar{a}_{2}[2 n]-\bar{a}_{2}[2 n-1]$. Then, IBI will be computed from $\Delta \bar{a}_{1}, \Delta \bar{a}_{2}$ and from the symbols $\left[s_{2 n-1} s_{2 n}\right]^{t}$ associated with each branch as $\bar{I}_{n}=0.5 \Delta \bar{a}_{1}-\Delta \bar{a}_{2} s_{2 n-1}^{*} s_{2 n}$ and $\bar{I} 2_{n}=0.5 \Delta \bar{a}_{2}-\Delta \bar{a}_{1} s_{2 n-1} s_{2 n}^{*}$. To cancel out IBI, the branch metric (8) is modified as

$$
\begin{aligned}
& \Lambda_{I C}\left(\Gamma_{n}, \boldsymbol{d}_{n+1}\right)=\| \boldsymbol{D}_{n+1}^{H} \boldsymbol{r}_{n+1}-\overline{\boldsymbol{I}}_{n+1}-\sum_{k=1}^{Q} b_{k}^{Q} . \\
& \left(\boldsymbol{D}_{n+1-k}^{H} \boldsymbol{r}_{n+1-k}-\overline{\boldsymbol{I}}_{n+1-k}\right) \|^{2}
\end{aligned}
$$

where $\overline{\boldsymbol{I}}_{n}=\left[\begin{array}{ll}\bar{I} 1_{n} & \bar{I}_{n}\end{array}\right]^{t}$. Then, Viterbi algorithm finds the sequence that minimizes

$$
l(\boldsymbol{r} \mid \boldsymbol{s})=\sum_{n=0}^{N-2} \Lambda_{I C}\left(\Gamma_{n}, \boldsymbol{d}_{n+1}\right) .
$$

This operation can be iterated further in later stages. However, the performance gain achieved by two iterations are most distinct as will be seen in the next section. The receiver described above will be noted as multistage receiver (MR).

We can approximate additional complexity of MR compared to VR for each trellis interval as follows. Suppose DSTBC applies $M$-ary constellation. Let $N_{s}$ be number of iterations (stages) and $N_{t a p}$ be the number of taps of the interpolation filter. To obtain $\Delta \bar{a}_{1}$ or $\Delta \bar{a}_{1}$, each requires $N_{\text {tap }} / 2$ multiplications, $N_{\text {tap }} / 2-1$ additions and 1 subtraction. To obtain $\bar{I} 1$ or $\overline{I 2}$, each requires 3 multiplications and 1 subtraction for each combination of transmitted symbols in a block. For each branch metric, it requires additional $2(Q+1)$ subtraction. In summary, MR requires additional $\left(N_{t a p}+6 M^{2}\right)\left(N_{s}-1\right)$ multiplications and $\left(N_{\text {tap }}+M^{2}+2(Q+1) M^{2 P}\right)\left(N_{s}-1\right)$ additions/subtractions for each trellis interval compared to VR.

\section{Vi. Simulation Results and Discussion}

We consider two fading scenarios when fading rates are $f_{d} T=0.02$ and $f_{d} T=0.05$. With MR, the interpolation filter is chosen to be a raised-cosine filter with roll-off factor $\alpha=0.2$. The cutoff frequency of the filter is chosen to be some amount higher than maximum Doppler frequency to avoid cutting high amplitude spectrum at the edge. The cutoff frequency for $f_{d} T=0.02$ is 0.0275 and for $f_{d} T=0.05$ is 0.075 . The number of filter taps is 100 for $f_{d} T=0.02$ and is 80 for $f_{d} T=0.05$. They are chosen to have enough taps such that they retain raised-cosine power spectrum. The parameters $P$ and $Q$ are one and five as in [5]. The number of iterations are chosen to be two because it yields maximum improvement.

In Fig. 2, the BER performance of DSTBC with MR is compared to CR, and VR, without coding scheme. From SNR $=0 \mathrm{~dB}$ to $\mathrm{SNR}=25 \mathrm{~dB}$, we design optimal prediction filter at each SNR. However, when SNR is greater than $25 \mathrm{~dB}$, we encounter increasing BER while increasing SNR. This happens from the assumption of fixed channel gain in the prediction filter design. To avoid this phenomenon, we use a prediction

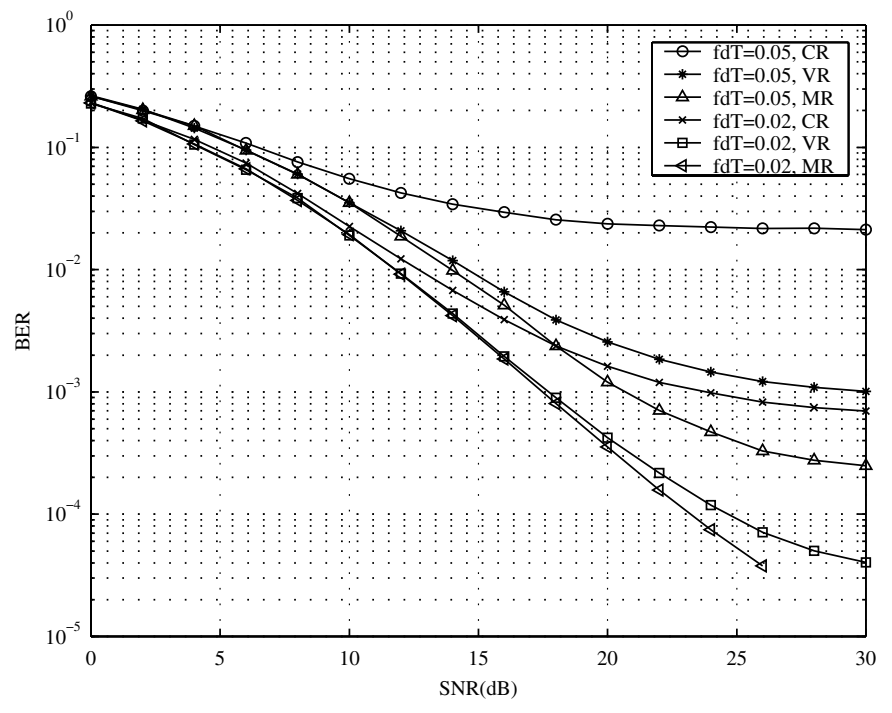

Fig. 2. Performance of DSTBC with BPSK, at fading rate 0.02 and 0.05 with $\mathrm{CR}, \mathrm{VR}$, and MR two iterations

filter designed at $\mathrm{SNR}=20 \mathrm{~dB}$ for channel SNR values greater than $20 \mathrm{~dB}$. From the figure, we can see that although VR reduces the error floor associated with CR significantly, it still has some amount of error floor due to IBI, especially at $f_{d} T=0.05$. At this fading rate, MR outperforms VR for SNR greater than $12 \mathrm{~dB}$ when the channel estimate from the first iteration becomes more reliable. Nevertheless, at $f_{d} T=0.02$, MR outperforms VR for SNR greater than $16 \mathrm{~dB}$ because IBI has less effect at this fading rate. Therefore, MR can further reduce the error floor with only two iterations. However, as the number of iterations is greater than two, MR provides virtually no improvement.

Fig. 3 compares the error floor of $\mathrm{CR}, \mathrm{VR}$ and MR evaluated at SNR $=30 \mathrm{~dB}$. It is seen that MR significantly reduces the error floor from VR and CR. MR can achieve about halforder of magnitude error floor improvement over VR from $f_{d} T=0.02$ to $f_{d} T=0.07$.

Fig. 4 shows the BER performance of $\mathrm{CR}$, VR, and MR in a coded system without interleaver. A rate $1 / 2$, convolutional code with constraint length $=5$, proposed in EDGE system is chosen [11]. The generator matrix is $\left[1+D^{3}+D^{4} 1+D+D^{3}+D^{4}\right]$. The overall transmission rate is $0.5 \mathrm{bit} / \mathrm{sec} / \mathrm{Hz}$. It can be seen that the convolutional code improves the BER performance of CR and VR significantly. At $f_{d} T=0.05$, MR outperforms VR after SNR $=12$ $\mathrm{dB}$ with two iterations. Significant gain can still be achieved at higher SNR with MR. However, at $f_{d} T=0.02$, there is virtually no improvement using MR or VR within this SNR region. One reason for this is, at this fading rate, the amount of IBI is small such that the error bits caused by IBI can be corrected by the powerful convolutional code decoder. Another reason is that the operating SNR is not high enough for error floor to appear. One might ask if we can use the tentative decision output from the convolutional decoder and recode it to DSTBC to improve the reliability of channel estimate for 


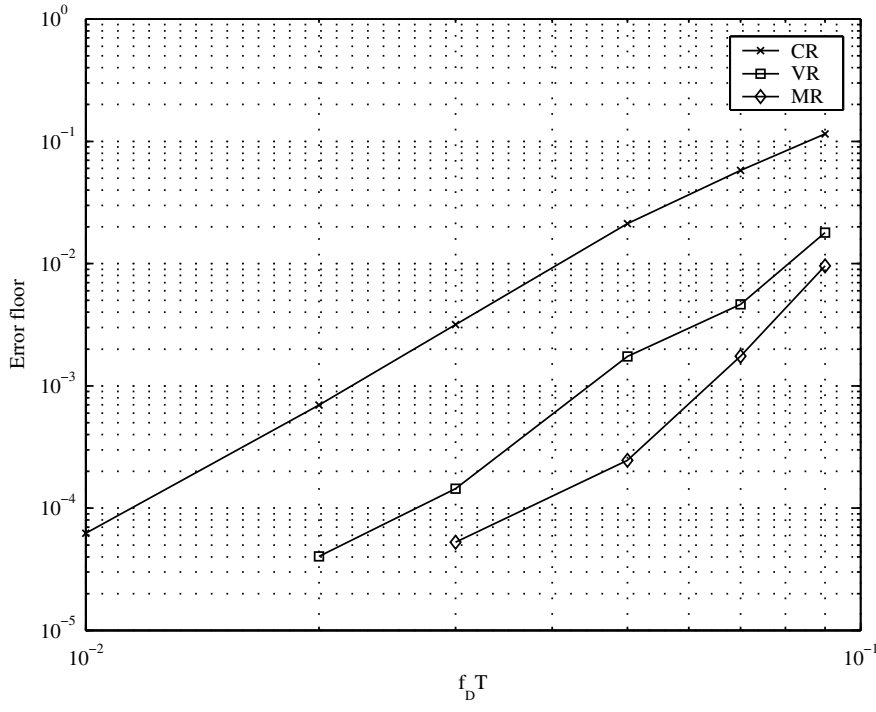

Fig. 3. Error floor of DSTBC with CR, VR, and MR evaluated at SNR = $30 \mathrm{~dB}$

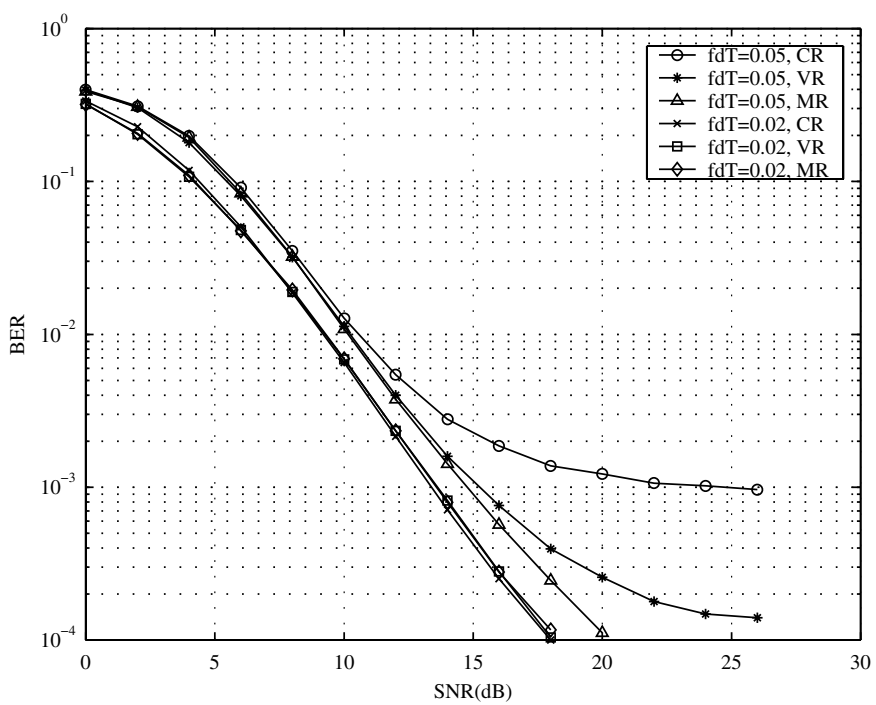

Fig. 4. Performance of convolutional code - DSTBC with BPSK, at fading rate 0.02 and 0.05 with $\mathrm{CR}, \mathrm{VR}$, and $\mathrm{MR}$ two iterations

the second iteration. However, it is found from our simulations (not shown) that using tentative decision from convolutional decoder does not have visible gain over typical MR within this SNR region while increasing complexity.

\section{CONCLUSIONS}

Multistage receiver for Tarokh's differential space-time block codes is proposed in this paper. It relaxes the assumption of fading behavior in previous detectors by taking channel time variation within a transmission block into account. With some additional complexity, multistage receiver provides further improvement over an approximate ML receiver. The proposed approach using only two iterations reduces the error floor by about half order of magnitude provided by approximate ML receiver for normalized fading rate between 0.02 and 0.07 in the uncoded system. Improvement can also be seen in the coded system at high fading rate.

\section{ACKNOWLEDGMENT}

The first author would like to thank Eugenio Chiavaccini for some discussions and Prof. Giorgio M. Vitetta for a preprint of their paper as well as anonymous reviewers. This research was supported by Natural Sciences and Engineering Research Council (NSERC) under their Strategic project grant program.

\section{REFERENCES}

[1] S.M. Alamouti, "A simple transmitter diversity scheme for wireless communications," IEEE J. Select. Areas Commun., vol. 16, no. 8, pp. 1451-1458, Oct. 1998.

[2] V. Tarokh and H. Jafarkhani, "A differential detection scheme for transmit diversity," IEEE J. Select. Areas Commun., vol. 18, no. 7, pp. 1169-1174, July 2000.

[3] B.L. Hughes, "Differential space-time modulation," IEEE Trans. Inform. Theory, vol. 46, no. 7, pp. 2567-2578, Nov. 2000.

[4] B.M. Hochwald and W. Sweldens, "Differential unitary space-time modulation," IEEE Trans. Commun., vol. 48, no. 12, pp. 2041-2052, Dec. 2000.

[5] E. Chiavaccini and G.M. Vitetta, "Further results on Tarokh's space-time differential technique," in Proc. IEEE ICC 2002, vol. 3, pp. 1778-1782, 28 Apr.-2 May 2002

[6] B.H. Park, K.J. Kim, S.-Y. Kwon and K.C. Whang, "Multistage decisiondirected channel estimation scheme for DS-CDMA system with M-ary orthogonal signaling," IEEE Trans. Veh. Tech., vol. 49, no. 1, pp. 43-49, Jan. 2000.

[7] R. Schober and L.H.-J. Lampe, "Noncoherent receivers for differential space-time modulation," IEEE Trans. Commun., vol. 50, no. 5, pp. 768777, May 2002.

[8] C. Ling and $\mathrm{X}$. Wu, "Linear prediction receiver for differential spacetime modulation over time-correlated Rayleigh fading channels," in Proc. IEEE ICC 2002, vol. 3, pp. 788-791, 28 Apr.-2 May 2002.

[9] J.H. Lodge and M.J. Moher, "Maximum likelihood sequence estimation of CPM signals transmitted over Rayleigh flat-fading channels," IEEE Trans. Commun., vol. 38, pp. 787-794, June 1990.

[10] G.M. Vitetta and D.P. Taylor, "Maximum likelihood decoding of uncoded and coded PSK signal sequences transmitted over Rayleigh flatfading channels," IEEE Trans. Commun., vol. 43, no. 11, pp. 2750-2758, Nov. 1995.

[11] ETSI, "Digital cellular telecommunications system (Phase 2+); channel coding," ETSI TS 100 909, v8.6.0, (2000-11).

[12] G.H. Golub and C.F. Van Loan, Matrix Computations. The John Hopkins University Press, 1989. 